\title{
Equal, Similar, but Different: Convergent Bonobos and Conserved Chimpanzees
}

\section{Citation}

Hare, B., and R. W. Wrangham. 2017. Equal, Similar, but Different: Convergent Bonobos and Conserved Chimpanzees. In Chimpanzees and human evolution, ed. M. N. Muller, R. W.

Wrangham, \& D. R. Pilbeam, 142-173. Cambridge, MA, US: Belknap Press of Harvard University Press.

\section{Permanent link}

http://nrs.harvard.edu/urn-3:HUL.InstRepos:42656533

\section{Terms of Use}

This article was downloaded from Harvard University's DASH repository, and is made available under the terms and conditions applicable to Open Access Policy Articles, as set forth at http:// nrs.harvard.edu/urn-3:HUL.InstRepos:dash.current.terms-of-use\#OAP

\section{Share Your Story}

The Harvard community has made this article openly available. Please share how this access benefits you. Submit a story.

Accessibility 
6 Equal, similar but different: convergent bonobos and conserved chimpanzees

Brian Hare ${ }^{1,2}$ and Richard Wrangham ${ }^{3}$

13

14

15

16

17

18

19 1Department of Evolutionary Anthropology, Duke University

20

212 Center for Cognitive Neuroscience, Duke University

22

$23{ }^{3}$ Department of Human Evolutionary Biology, Harvard University

24

25

26

27 


\section{Abstract}

29 Humans are lucky to have two closest relatives and not just one. While chimpanzees

30 have been the focus of far more research and are thought to more closely represent

31 our last common ape ancestor, the bonobo will be as important as the chimpanzee

32 in reconstructing our past. Understanding human evolution requires not only

33 knowing what traits are derived but also identifying what process may have shaped

34 these traits as they evolved. We suggest in this paper that while chimpanzees may

35 be best suited to help identify derived traits, bonobos will be crucial to testing ideas

36 regarding how selection shaped the human lineage. We base our argument on the

37 self-domestication hypothesis that has generated a host of recent studies comparing

38 the development of morphology, physiology, behavior and cognition in bonobos and

39 chimpanzees.

40 
"If the deduction that the bonobo is highly derived with respect to the other chimpanzees is correct, then its similarities to humans become even more significant: the original human environment has long been exceeded, but we may suppose that bonobos still occupy the niche to which they are best adapted. Thus by examining this niche we may learn something about the early human niche,

\section{Introduction}

50 A critical goal for human evolutionary biology is understanding when and how traits

51 evolved in our ancestral lineage during the 5-7 million years since our split with the

52 ancestors of chimpanzees and bonobos. Comparisons among humans and living

53 apes are essential in this task, because they allow us to discriminate between traits

54 that we share through common descent and those that have been modified since our

55 split from Pan. Central to interpreting these types of comparisons is determining

56 which living ape, if any, most closely resembles our last common ape ancestor or

57 "Pancestor" (Wrangham \& Pilbeam, 2001). Here we suggest based on the most

58 recent quantitative comparisons of behavior, physiology, development and

59 cognition that in many respects chimpanzees (Pan troglodytes) most closely

60 approximate our last common ancestor with chimpanzees and bonobos (our

61 "Pancestor"). We also suggest that bonobo (Pan paniscus) and human psychology

62 are similar in ways that indicate that cognitive evolution in the two species was

63 guided by parallel mechanisms (Hare et al, 2012).

64 Phylogenetic comparisons with both living and extinct apes are crucial for 65 reconstructing human evolution. Unfortunately cognition, temperament, personality

66 and all other psychological features do not fossilize. Nor have they typically been

67 linked to traits that can be measured in fossils. This means for the study of

68 psychological evolution our information is mostly restricted to comparisons of

69 humans with bonobos and chimpanzees, which fortunately provide informative

70 lessons. For example all three species readily follow the gaze of another individual

71 but only humans attribute false beliefs to others (e.g. Herrmann et al, 2010; 
72 Tomasello et al, 2007; Hare et al, 2001). We can conclude that the Pancestor used

73 gaze-following to predict and manipulate the behavior of others but it did not

74 understand that other could have false beliefs. The lack of false belief understanding

75 in the Pancestor makes sense, given that in humans the attribution of false beliefs is

76 tightly linked to language development. Unlike gaze following, therefore, attribution

77 of false beliefs is a derived feature of human evolution that evolved in the last 5-7

78 million years. Fossils cannot give us this level of resolution on questions of cognitive

79 evolution (Hare, 2011). When bonobos and chimpanzees share the same trait,

80 comparisons with humans are thus directly illuminating.

81 By contrast when bonobos and chimpanzees exhibit different traits a further

82 step is required to understand the evolutionary implications of the three-way

83 comparison. The problem is that when one of the two species is more similar to

84 humans the similarity can be due either to shared ancestry or to convergence, and it

85 is often not obvious which explanation is relevant. For example, direct quantitative

86 comparisons show that chimpanzees are better at causal reasoning while bonobos

87 are more skillful in social tasks (Herrmann et al, 2010). To understand the

88 implications of such observations for human evolution we need to know if the

89 relevant abilities evolved through shared ancestry or convergence.

\section{Two equal cousins both similar and different}

92 Robert Yerkes, the so-called father of American primatology (Haraway 1993), did

93 not realize that the infant bonobo and chimpanzee living with him for a year (i.e.

94 Chim and Panzee) were different species (de Waal \& Lanting, 1998). His failure is

95 understandable. The genomes of the two species differ by a mere $0.4 \%$ (Prufer et al,

96 2012), and the two species are in many ways very similar in their morphology,

97 feeding adaptations, cognition, life-history and social systems (Table 1).

98 Yet there are important differences that have persisted as long as $\sim 1$ million

99 years. Genomic comparisons indicate that, unlike other primates that show graded

100 hybridization zones during their early evolution, after isolation the two species did

101 not interbreed (Mailund et al, 2012). A sudden split between the proto-chimpanzees

102 and proto-bonobos is consistent with the Congo River creating a north-south barrier 
103 across the Congo Basin (since these apes do not swim) (Thompson, 2003). The lack

104 of gene flow allowed each species to evolve unique genetic and phenotypic features.

105 Comparisons to humans suggest that about $3 \%$ of the human genome is more

106 similar to either the bonobo or chimpanzee genome than the two sister species are

107 to each other, while about a quarter of human genes have some elements more

108 similar to one species of Pan over the other - but again in approximately equal

109 proportions (Prüfer et al, 2012). Behavioral and cognitive comparisons likewise

110 show striking differences between the two species of Pan, including many traits in

111 which one Pan species is markedly more like humans (Table 2, Hare et al, 2012;

112 Hare \& Yamamoto, 2015).

113 Given these profiles of overall similarities mixed with differences that vary

114 by species in their overlap with humans, how do we decide whether bonobos or

115 chimpanzees is more representative of the Pancestor with respect to any particular

116 trait? Three main hypotheses have been suggested:

118 1) The Mosaic hypothesis: No living ape is more representative. The

119 Pancestor's traits represented a mosaic of those found among bonobos and

120 chimpanzees (Prufer et al, 2012).

121 2) The Bonobo-like hypothesis: The Pancestor most closely resembled

122 bonobos (Zihlman, 1978; de Waal \& Lanting, 1998).

123 3) The Chimpanzee-like hypothesis: The Pancestor most closely resembled

124 chimpanzees (Wrangham \& Pilbeam, 2001).

125

126 The mosaic hypothesis claims that the relationship between traits in the

127 living apes is not sufficiently consistent to suggest that one species is more

128 representative of Pancestor over the others. It thus represents a null hypothesis.

129 Prufer et al. (2012) seem to suggest that comparisons of the bonobo, chimpanzee

130 and human genomes support this hypothesis, because they did not find any patterns

131 indicating that one species of Pan has been under stronger selection or is more

132 similar to humans than the other. In support, Table 2 show that specific behavioral 
133 and cognitive traits are found for which each species of Pan is more similar to

134 humans than to the other (Hare \& Yamamoto, 2015).

135 The bonobo-like hypothesis has been suggested based partly on qualitative

136 comparisons of behavior and cognition. For example within-group relationships

137 show similar trends in tolerance between bonobos and humans (de Waal and

138 Lanting, 1998). Morphological similarities between bonobos and australopithecenes

139 have also been used as a basis for inferring behavioral similarities between bonobos

140 and a pre-human stage of the ancestral human lineage (Zilhmann, 1978). The

141 bonobo-like hypothesis has not yet been carefully elaborated however. In particular

142 no conceptual scheme has been proposed that purports to account for patterns of

143 trait change in bonobos, chimpanzees and humans.

144 The chimpanzee-like hypothesis conforms to evidence that bonobos are

145 highly derived phenotypically relative to other nonhuman apes (Wrangham \&

146 Pilbeam, 2001). This hypothesis has led to quantitative tests that examine its core

147 developmental and cognitive predictions (Hare et al, 2012; Wilkins et al, 2014).

148 Below we review the hypothesis and the research that was designed to test it. We

149 also highlight how this recent work contradicts the predictions of the two preceding

150 alternative hypotheses. We conclude by suggesting where these hypotheses must be

151 tested further.

152

153 Bonobos and chimpanzees behaving social

154 Both bonobos and chimpanzees are highly social, knuckle-walking, fruit-eating,

155 large-brained apes. They live in fission-fusion communities composed of multiple

156 breeding females and males, potentially including more than 100 individuals. They

157 form friendships, groom, fight and reconcile within their groups. Males of both

158 species are larger than females and females have sexual swellings and mate

159 promiscuously. In both species females tend to emigrate and males are philopatric

160 (Kano, 1992; Boesch et al, 2002; Muller \& Mitani, 2005). Given the similarities

161 between the two species, differences become more striking. Ethologists first began

162 to notice these differences in the earliest studies of their social behavior -

163 particularly involving the expression of aggression. Chimpanzees are characterized 
164 by clear dominance hierarchies within groups and hostile relationships between 165 groups. Males are dominant, coerce females, commit infanticide, strive for alpha 166 status, are highly territorial, xenophobic and display coalitionary aggression that 167 can be lethal (Muller \& Mitani, 2005; Wrangham et al, 2006; Muller et al, 2011;

168 Wilson et al, 2014; Feldblum et al, 2015). Chimpanzee mothers support their 169 offspring and are aggressive toward immigrants. They may form friendships with 170 other mothers that can be used in aggressive coalitions, but alliance flexibility has 171 not been reported (Pusey et al, 2008; 2013; Kahlenberg et al. 2008). In contrast 172 bonobos show much less intense forms of aggression within and between their 173 groups. Bonobo males do strive for status, but do not form coalitions with other 174 males or attain alpha status within their own groups (Kano, 1992). Mothers are 175 typically a male's most important social partner - with grandmothers potentially 176 impacting male social status (Surbeck et al, 2011; Schubert et al, 2013). Bonobo

177 females do form coalitions with each other and are intolerant of male coercion 178 (Kano, 1992; Wrangham, 2002; Hohmann \& Fruth, 2003). Bonobos do not show the 179 pattern of lethal aggression observed in chimpanzees and even have been seen to 180 socially interact and travel with neighboring groups (Furuichi et al, 2012). Female 181 immigrants are prized social partners for resident female bonobos and they receive 182 intense attention but relatively little physical aggression (Sakamaki et al, 2015; Ryu 183 et al, 2015; Moscovice et al, 2015).

184 These stark differences in social behaviors related to aggression and 185 affiliation again illustrate what is at stake in more precisely estimating which 186 species is more representative of Pancestor. One set of explanations and tests will

187 be necessary to reconstruct human evolution if Pancestor was a male dominated, 188 xenophobic species and a totally different set if it was a more female-centered, 189 relatively xenophilic species. A focus on this contrast leads to a unifying explanation 190 for how these traits evolved.

\section{Process matters: the evolution of development}

193 Bonobos were the last great ape discovered, first recognized in a museum. A small

194 skull labeled as "juvenile" was inside a box that on inspection had fused cranial 
195 sutures. This suggested the skull was adult and must belong to a kind of chimpanzee

196 that was different from Pan troglodytes (Schultz 1929; Coolidge, 1933). The

197 developmental difference led not only to the identification of bonobos but also to

198 the chimpanzee-like hypothesis.

199 Because ontogenetic stages are temporary they are often given less attention

200 than adult forms. However changes in the timing of development provide natural

201 selection with one of the riches sources of variation to act on (West-Eberhard,

202 2003). Relatively small changes in developmental trajectories can have large effects

203 on adult phenotypes, and in many cases selection acts strongest early in

204 development (West-Eberhard, 2003; Hill et al, 2001; Morimoto et al, 2014).

205 Furthermore changes in seemingly unrelated traits may be explained through a

206 relationship formed via a common developmental pathway. Comparative analysis of

207 development can therefore provide a powerful way to understand species

208 differences.

209 While it may not be widely appreciated that bonobos were recognized as a

210 species based on their skulls resembling those of juvenile chimpanzees, they are

211 well known for having high levels of play and diverse patterns of sexual behavior in

212 adulthood (Palagi \& Cordoni, 2012). These behavioral tendencies are unique

213 compared to other apes, and exemplify a suite of bonobo traits that share two

214 intriguing features. First, their expression in adult bonobos resembles their

215 expression in juvenile chimpanzees. Second, their differences in expression between

216 bonobos and chimpanzees resemble the differences in expression between

217 domesticated animals and their wild ancestors (Wrangham \& Pilbeam, 2001; Hare

218 et al, 2012; Wilkins et al, 2014). Thus like bonobos compared to chimpanzees,

219 domesticated animals have reductions in the size of crania, faces and teeth, together

220 with a variety of novel prosocial behaviors and accompanying physiological

221 changes, compared to the adult forms of their wild progenitors. These observations

222 suggest that the behavioral, physiological and morphological changes observed both

223 in bonobos and in domesticated animals are strongly influenced by heterochronic

224 change. According to this idea, selection for a reduction in aggression is the driving

225 force in generating a syndrome of traits associated with domestication. The 
226 domestication syndrome results from selection favoring individuals who retain 227 juvenile characteristic into adulthood, including a reduced propensity for reactive 228 aggression compared to the ancestral adult (Trut et al, 2009).

Following the logic developed for domesticated animals, Wrangham and 230 Pilbeam (2001) therefore proposed that bonobo behavioral evolution is explicable

231 by considering development. As with the initial discovery of bonobos, they began 232 with morphology. They noted that while gorillas and chimpanzees share the same 233 ontogenetic pattern for the trajectory of both their head and body, in bonobos the 234 head is relatively small. They pointed out qualitative similarities between the 235 recurring pattern of changes observed between domesticated and wild progenitors 236 across species and the differences seen in bonobos and chimpanzees. They

237 hypothesized that the observed convergence in traits of the "domesticatioan

238 syndrome" between bonobos and domesticated animals is a result of socio-

239 ecological pressures that selected bonobos to be less aggressive, as in domesticated

240 animals. The implication was that bonobos evolved from a chimpanzee-like

241 Pancestor when they were isolated on the left bank of the Congo River, thanks to

242 selection for reduced aggression. Essentially, the list of qualitative differences listed

243 in Table 2 that seem to suggest a mosaic pattern of evolution began to make sense

244 using development as the organizing principle for their origin.

\section{The psychology of bonobo self-domestication}

247 Recently Hare et al (2012) formalized the "self-domestication" hypothesis (SDH) for 248 bonobo evolution and reviewed the body of work testing the main predictions from 249 Wrangham and Pilbeam (2001). The SDH hypothesis relies heavily on the work of 250 Dmitry Belyaev and colleagues who experimentally selected a population of silver 251 foxes (Vulpes vulpes) to be interested and prosocial towards humans as opposed to 252 aggressive and fearful toward them. The experimental line of foxes was compared

253 to a line of control foxes selected randomly for their behavior toward humans.

254 Otherwise the two fox populations were maintained identically. When compared to 255 the control line of foxes the experimental foxes showed the majority of 256 physiological, morphological and behavioral elements observed in the 
257 domestication syndrome seen in most domesticated animals. This included

258 feminized crania, smaller teeth, floppy ears, shortened tails, more play and sexual

259 behavior as adults as well as prosocial behavior toward humans. The experimental

260 foxes also became more skilled at using human gestures similar to the way dogs are

261 more skilled than wolves - even though the foxes were not selected based on their

262 communicative abilities (Hare et al, 2005). Developmental comparisons of the two

263 populations suggest that many of the differences are a result of heterochrony (cf.

264 Gariepy et al, 2001). For example, the experimental foxes have extended

265 socialization periods (Trut, 1999). Selection against aggression and for prosociality

266 thus appears to have acted on individual differences in developmental timing to

267 produce a population of foxes that has not only become attracted to humans but also

268 shows a suite of correlated by-products, from morphology to social cognition, none

269 of which were under direct selection (Trut, 2009).

270 The silver-fox experiment reveals the power of selection for prosocial

271 behavior on morphology, behavior, physiology and even cognition. It also suggests

272 the possibility that natural selection acting on wild animal populations could drive a

273 similar type of "self-domestication" (Hare et al, 2012). Because of the qualitative

274 similarities documented between bonobos and domestic animals, quantitative tests

275 were designed to examine the hypothesis that bonobos are self-domesticated. The

276 majority of these a priori quantitative tests of the SDH involve comparing bonobo

277 and chimpanzee cognition since the SDH was largely proposed based on

278 observational studies that lead to clear predictions about where cognitive

279 differences should evolve in response to selection against aggression (Wrangham \&

280 Pilbeam, 2001; Hare et al, 2012). Key cognitive predictions were that bonobos

281 should show greater prosociality than chimpanzees, as well as more flexible social

282 skills relating to cooperation and communication - similar to what has been

283 documented when comparing cognition between wild types and domesticated

284 animals (e.g. Hare et al, 2002; 2010; Miklosi et al, 2003; Topa et al, 2009; Hernadi et

285 al, 2012). Furthermore, if bonobos are juvenilized as a result of their reduced

286 aggression being due to heterochronic change, they should show delays in their 
287 cognitive and behavioral development relative to chimpanzees (Wobber et al, 288 2010).

\section{Prosocial bonobos prefer strangers}

291 The most obvious behavioral novelties common to all domestic animals are an

292 increase in prosociality and reduction in the propensity for aggression. Specifically

293 domesticated animals are attracted to and interested in interacting with humans

294 while their wild type ancestors are fearful or even aggressive towards people (Hare

295 et al, 2012). Similarly bonobos are more prosocial to conspecifics than chimpanzees.

296 By contrast natural selection did not act on their reaction to humans (Tan \& Hare, in 297 prep.).

298 The relative increase in prosociality among bonobos is illustrated by the fact 299 that compared to chimpanzees they co-feed more readily. Thus when two bonobos

300 are released into a room containing food in a single location, both tend to be able to

301 eat. By contrast chimpanzees under the same conditions rarely co-feed because the

302 dominant normally monopolizes the food. This result holds even for chimpanzees

303 chosen for having a highly tolerant relationship with each other, whereas almost

304 any pairs of bonobo successfully co-feed. The high level of tolerance shown by

305 bonobos allows them to cooperate more effectively than chimpanzees, even when

306 chimpanzees are more experienced in the task (Hare et al, 2007). The different

307 responses of the two species are in part hormonally mediated. In tests of food-

308 sharing, male bonobos show an increase in cortisol (but not testosterone) in

309 anticipation of entering the room containing food, whereas male chimpanzees show

310 an increase in testosterone (but not cortisol). The hormone differences thus

311 indicate that in response to the same socially stressful condition, bonobos become

312 socially anxious while chimpanzees prime themselves for competition (Wobber et

313 al, 2010).

314 Parallel kinds of species difference in behavior occur in response to

315 crowding. In crowded enclosures of captive groups bonobos increase socio-sexual

316 and grooming behavior, whereas chimpanzees avoid aggression by decreasing

317 social interactions (Aureli \& deWaal, 1997; Paoli et al., 2007; Hare et al, 2007). Thus 
318 bonobos appear to use social interactions to diffuse tension while chimpanzees

319 must inhibit social interactions to accomplish the same outcome.

320 Like chimpanzees, bonobos sometimes share food in the wild (Yamamoto,

321 2015). Experiments in captivity help understand the motivation and cognition

322 behind this type of sharing. Chimpanzees share proactively only when they

323 themselves cannot access the food (Warneken et al, 2007; Melis et al, 2010).

324 However bonobos share not only when they cannot reach the food, but also when, if

325 they wanted, they could keep it all for themselves. For example one experiment

326 allows bonobos to voluntarily share food by opening a one-way door to release

327 another bonobo into the room with them. Bonobos with food repeatedly open the

328 door even when the other bonobo does not beg for food and has no opportunity to

329 exhibit reciprocal generosity (Hare \& Kwetuenda, 2010). Strikingly, bonobos will

330 open the one-way door to share food with a stranger - a bonobo from a different

331 social community. Even more remarkable, when given the choice of proactively

332 sharing with a stranger or groupmate bonobos prefer to share with a stranger (Tan

333 \& Hare, 2013; Bullinger et al, 2013; Tan et al, submitted). The sharing tendencies of

334 bonobos thus conform to the expectations of the self-domestication hypothesis by

335 showing that there is a marked increase in prosociality in bonobos compared to

336 chimpanzees.

337 Despite the unusual generosity of bonobos, it is important to note that their

338 sharing is also constrained. Similar to chimpanzees bonobos have not been

339 observed to share monopolizable food that is in their possession unless their

340 sharing leads to an affiliative social interaction (Jaeggi et al, 2010; Tan \& Hare, 2013;

341 Tan et al, 2014). In addition, while chimpanzees readily share objects upon request,

342 bonobos prefer to share food over objects (Warneken et al, 2007; Yamamoto et al,

343 2012; Krupenye et al, submitted).

344

\section{Prosocial temperament leads to social cognition}

346 An important cognitive result of domestication is more flexible cooperative-

347 communication (Hare, 2011; although also see Lewejohann et al, 2010). Dogs are

348 more skilled at spontaneously understanding human gestures than wolves (Hare et 
349 al, 2002; Hare et al, 2010). Dogs show similar flexibility in reading human pointing

350 gestures as seen in human infants (Hare \& Tomasello, 2005). Dogs are also much

351 more likely to make eye contact with a human than a wolf when faced with an

352 unsolvable task while also making socially mediated errors only seen in human

353 infants (Miklosi et al, 2003; Topal et al, 2009). Exogenous oxytocin also may

354 enhance the ability of dogs to use human gestures (Oliva et al, 2015). Dogs' unusual

355 skill at using human gestures has been attributed to selection against aggression

356 and for more prosocial behaviors toward humans. The strongest test of this idea

357 coming from a comparison between Belaev's silver foxes (selected to be attracted to

358 and nonaggressive toward humans) and the control population bred randomly in

359 relation to their response to humans. The experimental line of foxes was as skilled at

360 using human gestures as dog puppies and more skilled than control foxes (Hare et

361 al, 2005). A similar relationship between temperament and social cognition has

362 been observed in a variety of domesticated and nondomesticated species (e.g. Petit

363 et al, 1992; Lewejohann et al, 2010; Hernandi et al, 2012, Michelleta \& Waller, 2012;

364 Brust \& Guenther, 2015; Bray et al, 2015).

365 The self-domestication hypothesis therefore predicts that bonobos will out-

366 perform chimpanzees in tasks involving cooperative communication. This

367 prediction has been upheld. When directly compared on a battery of temperamental

368 tests given to bonobos, chimpanzees, orangutans (Pongo pygmaeus) and humans,

369 bonobos are most similar to human children (who are shy and observant relative to

370 the other ape species) (Herrmann et al, 2011). Similarly across a wide range of

371 cognitive tasks bonobos are more sensitive than chimpanzees to social cues such as

372 gaze direction (Herrmann et al, 2010; but see Maclean \& Hare, 2013). Moreover,

373 when bonobos and chimpanzees are compared using an eye-tracker only bonobos

374 spend significant time examining the eyes of social stimuli such as conspecific and

375 human faces. By contrast chimpanzees spent the majority of each trial examining

376 the mouths of the same set of stimuli. The response of bonobos in this case is much

377 more similar to that observed in normally functioning children while the response

378 of the chimpanzees resembles that of people on the autistic spectrum (Kano et al

379 2015). In corroboration of this interpretation, the 2D4D ratios observed in 
chimpanzees suggest high neonatal androgen levels while bonobos suggest levels more similar to normally functioning humans. High neonatal androgen levels and concomitant 2D4D levels have been linked to autism spectrum disorder and aggressive tendencies in humans (McIntyre et al 2009; Liu et al, 2012). generation of direct comparisons strongly suggests that bonobos are even much more human-like than chimpanzees in terms of their sensitivity to subtle social cues. The mechanisms underlying these differences remain to be explored, but there are intriguing hints. A positive relationship between oxytocin and levels of mutual gaze in dogs suggest that the oxytocin system could be implicated in differences observed

390 in bonobos. Dogs who make more eye contact with their owners have the highest

391 levels of oxytocin as do their owners (Nagasawa et al, 2009; 2015; Romero et al 392 2014). When dogs are given oxytocin exogenously they make more eye contact and 393 their owner's oxytocin level increases (Nagasawa et al, 2015). Possibly the increased 394 prosociality of bonobos is in part mediated by a similar oxytocin feedback loop that

395 is less sensitive in chimpanzees. This idea is supported by previously observed

396 genetic differences in bonobos related to the expression of oxytocin and vasopressin 397 (Hammock and Young, 2005; Staes et al, 2015; see also Garai et al, 2014). However, 398 this idea remains largely untested. Variability in chimpanzee social behavior has 399 been linked to urinary oxytocin levels (Wittig et al, 2014) but no similar tests have 400 yet been conducted with bonobos.

\section{Juvenilization: a mechanism for change}

403 Heterochronic change remains the leading explanation for the domestication 404 syndrome (Wilkins et al, 2014). If correct, the domestication syndrome should be 405 accompanied by developmental shifts that lead to juvenile traits being preserved 406 into adulthood (Wobber et al, 2011). As expected, recent quantitative comparisons 407 reveal that cognition is developmentally delayed in bonobos relative to chimpanzees 408 (see Table 3 for summary). For example, given that infant bonobos (with 409 mothers) receive less aggression than infant chimpanzees, Wobber et al (2010) 410 predicted that bonobos would show a delay in the development of social inhibition. 
411 The premise was that it is crucial for an infant chimpanzee to quickly understand 412 who they can and cannot forage near to avoid aggression and injury, whereas the

413 high level of feeding tolerance towards infant bonobos makes this type of inhibition

414 less vital. In a series of cognitive tasks bonobos indeed showed delayed

415 development of social inhibition in comparison to age-matched chimpanzees.

416 Bonobos likewise showed evidence of developmental delay in spatial memory.

417 When a cross-sectional sample was compared, older chimpanzees showed

418 improvement in remembering where hidden treats were located in space while

419 bonobos did not: adult bonobos performed at levels similar to juvenile chimpanzees

420 (Rosati \& Hare, 2012).

421 Such differences might partly be hormonally mediated, given that male

422 bonobos have been reported to show no increase in androgens around puberty. By

423 contrast male chimpanzees exhibit the expected pubertal rise in androgens

424 (Wobber et al, 2014; although see Behringer et al, 2014). Furthermore individual

425 differences in chimpanzee androgen levels were strongly associated with

426 performance on the spatial navigation task, whereas androgen levels and spatial

427 cognition were not correlated in bonobos (Wobber \& Herrmann, 2015). Finally,

428 bonobos also show delayed offset of the expression of thyroid hormone relative to

429 chimpanzees, which might be linked to lower levels of aggression (Beringher et al,

430 2014). Other suspected cases of developmental delay include visual perspective

431 taking (Maclean \& Hare, 2012) and reputation formation (Herrmann et al, 2013).

432 Further experimentation will test these ideas.

433 Bonobos thus show delay in various behaviors and types of cognitive

434 performance relative to chimpanzees., whereas no cognitive task has yet been found

435 where chimpanzees show delay relative to bonobos. However sexual behavior

436 offers an exception to this overall pattern of species differences. Infant bonobos are

437 more sexually active than infant chimpanzees (Woods \& Hare, 2011), and female

438 bonobos appear to reach sexual maturity earlier than female chimpanzees

439 (Beringher et al, 2014; Ryu et al, 2015). Domesticated species similarly tend to

440 exhibit early reproduction (Wilkins et al, 2014). 
441 Given that the overall differences between bonobos and chimpanzees

442 represent a slower rate of development in bonobos, they could in theory have

443 resulted from either paedomorphism (slowed development in bonobos) or

444 peramorphism (accelerated development in chimpanzees). However peramorphism

445 in chimpanzees is an improbable mechanism because chimpanzee cranial

446 development is similar to gorillas Gorilla gorilla and other great apes (Wrangham \&

447 Pilbeam, 2001; Hare et al, 2012), whereas among the great apes bonobos are the

448 exception in having a relatively juvenilized cranium (Lieberman et al, 2007;

449 Durrleman et al, 2012). Since cognitive development is assumed to be causally

450 linked to the development of the brain and cranium, the evidence for bonobo crania

451 being paedomorphic supports the notion of bonobo cognition likewise being

452 paedomorphic, as expected by the self-domestication hypothesis.

$453 \quad$ Future work may show that the developmental predictions of the self-

454 domestication hypothesis can account for a variety of features in bonobos that are

455 difficult to explain as independent ecological adaptations, such as the retention of an

456 infantile white tail tuft into adulthood. Wilkins et al. (2014) recently proposed that

457 the domestication syndrome is the result of a delay in the migration of melanocytes

458 early in the development of the neural crest. These cells are responsible for the

459 formation of morphological and physiological features altered through

460 domestication (i.e. teeth, cartilage, melanin, etc.) as well as influencing brain

461 development through their effects on growth factors. Hare et al (2012) raise the

462 possibility that a similar developmental mechanism might account for the

463 convergence between bonobos and other domestic animals. The neural crest

464 hypothesis points to future tests that could reveal the heritable mechanism that was

465 affected to create the domestication syndrome across a wide variety of species,

466 including bonobos.

467

468 Cognitive support for reduced scramble competition

469 The main scenarios proposed to explain the behavioral divergence of bonobos and

470 chimpanzees are based on ecological difference (Wrangham, 1986; Furuichi, 2009).

471 Over approximately a million years of separation on either side of the Congo River, 
472 contrasts in ecology are posited to have shaped the two species into their modern 473 forms (Wrangham 1993, Furuichi 2009). Species-specific psychological

474 predispositions evolved accordingly, such that the social behavior of the two species

475 is now expected to differ even when they live in equivalent environments (Horuichi 476 2004, Serckx et al, 2015).

477 A critical species difference in social organization is that bonobo parties

478 (temporary sub-groups) are more stable than those in chimpanzees (Furuichi 479 2009). This gives bonobo mothers a predictable ability to form alliances with each 480 other and with their adult sons (Furuichi 2011). Such alliances deter aggression by 481 adult males, and are therefore inferred to have reduced the benefits to males of 482 having a high propensity for aggression (Wrangham 1993, Furuichi 2011).

483 Using current ecological differences as a proxy for past selection pressures, 484 the tendency for bonobos to form more stable parties is hypothesized to have 485 evolved in a context of reduced scramble competition for fallback foods compared to 486 chimpanzees. One possibility is that bonobo forests differ floristically from 487 chimpanzee forests. For example, bonobo forest might have larger fruit trees or a 488 higher density of food patches (Furuichi 2011). Against this, however, there is little 489 evidence for consistent differences in these respects between bonobo and 490 chimpanzee habitats (Sommer et al 2011).

491 Another possibility is that the reduction in feeding competition for bonobos 492 is due to the lack of gorillas within the species range of bonobos (Wrangham 1986, 493 Wrangham \& Peterson, 1996). Gorillas rely heavily on the stems and leaves of 494 terrestrial herbs for their food supply. These items are also eaten by chimpanzees 495 and bonobos as fallback foods when preferred fruits are less available. The absence 496 of gorillas south of the Congo River is therefore argued to afford bonobos a greater 497 reliance on terrestrial herbs as fallback foods. As a result, bonobos can forage 498 together in relatively stable parties, as occurs among gorillas. Novel quantitative 499 tests of this hypothesis remain difficult since there is no fossil record from the 500 Congo Basin to test the distribution of gorillas over evolutionary time, and cropping 501 rates of terrestrial herbs either side of the Congo River have not been studied in 502 detail. 
503 A cognitive approach can help in testing ecological predictions (Rosati et al,

504 2014). If bonobos and chimpanzees were shaped over the past by ecological

505 differences that mirror those thought to exist today, we should see species

506 differences in foraging strategies that reflect those differences. Previous

507 comparisons between closely related species illustrate such links among feeding

508 strategies, decision-making and spatial memory (Santos \& Rosati, 2015). For

509 example frugivorous lemurs are more successful than more folivorous lemur species

510 in a set of spatial memory tasks (Rosati et al., 2014). Likewise in a stationary context

511 insectivorous tamarins (Saguinus oedipus) prefer small immediate rewards whereas

512 gummivorous marmosets (Callithrix jacchus) prefer larger delayed rewards (Rosati

513 et al., 2006). However if travel is required to obtain the same rewards the monkeys

514 reverse their preferences (Stevens et al, 2005). Thus the feeding ecology to which a

515 species is adapted is expected to influence their foraging cognition.

516 Accordingly if consistent differences in the nature of the Pleistocene food

517 supply shaped bonobo and chimpanzee social tendencies, they are expected to have

518 influenced their cognitive preferences also (Hare et al, 2012). To test for this

519 possibility bonobos and chimpanzees have been quantitatively compared across a

520 range of foraging tasks designed to measure how they remember locations and

521 value time and risk. Compared to bonobos, chimpanzees tend to prefer larger

522 rewards, and they tolerate longer delays in waiting for them(Rosati et al, 2007;

523 Rosati \& Hare, 2013). Similarly in assessments of preference for a fixed or variable

524 reward, bonobos prefer a small fixed reward while chimpanzees prefer the option of

525 a more risky, but potentially larger variable reward (Heilbronner et al, 2008; Rosati

526 \& Hare, 2014; see also Haun et al, 2011). Memory tasks reveal parallel results:

527 chimpanzees tend to outperform bonobos by finding more hidden food items, across

528 longer delays (Rosati \& Hare, 2012; Rosati, 2015). These differences between

529 bonobos and chimpanzees thus conform to the ecological model of reduced

530 scramble competition. They may again be mediated by androgens since spatial

531 performance was linked to basal testosterone levels in chimpanzees but not in

532 bonobos (Rosati, 2015; Wobber \& Herrmann, 2015). 
533 Overall, therefore, research on developmental and cognitive comparisons

534 between bonobos and chimpanzees tends to support the self-domestication

535 hypothesis. Bonobos show a suite of traits that are seen in a range of species that

536 have undergone juvenilization in the course of selection against aggression.

537 Cognitive comparisons support the idea that this process occurred due to natural

538 selection caused by reduced scramble competition. Neither the mosaic nor the

539 bonobo-like ancestor model predict or can explain the range of developmental

540 differences reviewed here. Chimpanzees likely serve as the better living

541 representative of the Pancestor, while the more derived bonobo gives us a powerful

542 way to think about the evolution of the most derived ape of all.

543

\section{Human self-domestication}

545 It has been repeatedly suggested that humans are domesticated. Stephen J.

546 Gould famously argued that humans are globally paedomorphic with this

547 developmental pattern being crucial to our species' evolution (Gould, 1980). While

548 the idea of humans being globally juvenilized has been rejected, the idea that

549 humans are domesticated has gained new life (e.g. Leach, 2003). One of the main

550 impetus for this is the comparative work explored above. Observing the constraints

551 on chimpanzee cooperation and the unusual social skills of dogs led to the

552 suggestion that temperamental evolution is a powerful mechanism to shift social

553 problem solving abilities (Hare \& Tomasello, 2005; Hare et al, 2007; 2011; Bray et

554 al, 2015). In support of this idea children with shy temperament tend to develop

555 sophisticated theory of mind skills earlier in development. This provides the first

556 direct evidence that even in humans there may be an important link between

557 temperament and social cognition that is crucial for unique forms of human

558 cognition (Wellman et al 2012; Lane et al 2013; also see Rodrigues et al 2009).

559 Direct comparisons of cognitive development across a range of cognitive tasks in

560 bonobo, chimpanzee and human infants have also revealed that human infants have

561 a set of early emerging social cognitive abilities. These same abilities are thought to

562 be the cognitive foundation for participating in human culture (Herrmann et al,

563 2007). These findings support the idea that a shift in development led to the human 
564 condition (Wobber et al, 2014). Finally, there is even evidence from comparisons of

565 fossil humans that late in our species evolution we underwent selection against

566 aggression that may have not only shaped our morphology but more importantly

567 our whole way of life (Nelson et al, 2011; Cieri et al, 2014). Initial comparisons of

568 gene expression development in humans and chimpanzees also suggests

569 heterochronic shifts. Many genes that are switched off early in the development of

570 the chimpanzee prefrontal cortext are still switched on decades later in humans

571 (Somel et al, 2009). This initial work suggests that developing predictions for a new

572 generation of tests to examine human domestication will be an exciting area of

573 research in coming years and central to any test will likely be our more derived and

574 potentially convergent relative the bonobo.

575

576 Past and future limitations

577 While a first generation of experimental studies offers support for the main 578 cognitive predictions regarding the self-domestication hypothesis, not every study 579 comparing cognition in the two species of Pan has revealed differences. Many of the 580 similarities are observed in cognitive skills not predicted by the SDH to be altered.

581 For example, bonobos and chimpanzees perform similarly on nonsocial inhibitory

582 control in detour-reaching tasks (Vlammings et al, 2009; Maclean et al, 2014). The

583 two species also both use play and grooming as social currency in similar ways

584 (Schroepfer et al, 2015). Most significantly on a standardized battery of cognitive

585 tasks bonobos and chimpanzees were identical in most ways except in areas that are

586 predicted by the self-domestication hypothesis (i.e. bonobos being more skilled with

587 social cues and chimpanzees being more skilled in some nonsocial tasks; Herrman et 588 al, 2010).

589 However, there have also been studies that predicted species differences but

590 did not detect them. Although it was expected that bonobos would be more averse

591 than chimpanzees to choosing ambiguous options, the two species show equal

592 aversity to ambiguity (Rosati \& Hare, 2011; Krupenye et al, 2015). Likewise,

593 bonobos are no more interested in joint object play than chimpanzees, and are no

594 more skilled at using human gestures to find hidden food (Maclean \& Hare, 2013; 
595 Maclean \& Hare, 2015). These findings should refine the self-domestication

596 hypothesis and act as examples of how quantitative comparisons that powerfully

597 test the predictions of the SDH are now possible and can potentially allow for

598 falsification.

599 To date large-scale quantitative comparisons between bonobos and

600 chimpanzees have been conducted at African sanctuaries designed to rescue,

601 rehabilitate and release bushmeat orphans (i.e. $N>20$ per species). This raises the

602 issue of how representative these individuals might be given the acute trauma they

603 experience. Direct comparison of mother-reared and orphan bonobos and

604 chimpanzees showed no differences in cognition, behavior or hormonal profiles

605 (Wobber et al, 2011). Moreover, a number of successful releases of orphans back

606 into the wild have been conducted (including orphans from Lola ya Bonobo; Andre

607 et al, 2008). This suggests the populations involved in recent quantitative

608 comparisons are representative of the cognitive flexibility in the species to which

609 they belong.

610 The self-domestication hypothesis suggests tests that may help explain both

611 bonobo and human evolution. However, questions regarding evolutionary process

612 in our lineage remain difficult to address. A small number of living species within

613 the ape clade means comparison between apes typically lack quantitative power.

614 Biologists have developed sophisticated quantitative phylogenetic techniques to test

615 evolutionary hypotheses that are not possible using a handful of species (Maclean et

616 al, 2011). While this lack of power has made it difficult to resolve the extent to

617 which the different species of Pan resemble the last common ancestor, it also

618 highlights the strength of a broader comparative developmental approach that

619 generated the self-domestication hypothesis.

620

\section{Summary}

622 Where fossils are silent comparisons of the living apes are crucial to testing

623 evolutionary ideas regarding the origin of traits. Interpretations of these

624 comparisons rest heavily on which living ape is considered more representative of

625 our Pancestor. A priori morphological, physiological, behavioral, cognitive tests of 
626 the self-domestication hypothesis support the chimpanzee-like hypothesis.

627 Bonobos not only are more prosocial and flexible in their social skills but also show 628 signs of juvenilization across the phenotype. This developmental pattern suggests

629 that bonobos are more derived than chimpanzees from our last common ancestor, 630 in line with a similar conclusion based on morphology. If support for the self-

631 domestication hypothesis grows, it will not only clarify which traits are derived in

632 humans but also generate hypotheses regarding the process(es) that shaped these 633 traits. The self-domestication hypothesis has already led to a number of tests 634 regarding the evolution of human cooperation and communication as a result of 635 selection against within group aggression. This means both bonobos and 636 chimpanzees will be needed equally to continue unlocking the secrets of human 637 evolution.

638

\section{Acknowledgements}

640 We thank James Brooks and Eliot Cohen for help with our reference section. 641 
643 Andre, C., Kamate, C., Mbonzo, P., Morel, D., Hare, B., 2008. The conservation value of 644 Lola ya Bonobo sanctuary. Bonobos: Behavior, Ecology, and Conservation, $645 \quad 303-322$.

646 Aureli, F., DeWaal, F.B.M., 1997. Inhibition of social behavior in chimpanzees under 647 high-density conditions. American Journal of Primatology 41, 213-228.

648 Behringer, V., Deschner, T., Deimel, C., Stevens, J.M.G., Hohmann, G., 2014. Age649 related changes in urinary testosterone levels suggest differences in puberty $650 \quad$ onset and divergent life history strategies in bonobos and chimpanzees. Hormones and Behavior 66, 525-533.

652 Behringer, V., Deschner, T., Murtagh, R., Stevens, J.M.G., Hohmann, G., 2014. Age653 related changes in Thyroid hormone levels of bonobos and chimpanzees indicate heterochrony in development. Journal of Human Evolution 66, 8388.

Brust, V., Guenther, A., 2015. Domestication effects on behavioural traits and learning performance: comparing wild cavies to guinea pigs. Animal Cognition 18, 99-109.

662 Cieri, R.L., Churchill, S.E., Franciscus, R.G., Tan, J.Z., Hare, B., 2014. Craniofacial Feminization, Social Tolerance, and the Origins of Behavioral Modernity. Current Anthropology 55, 419-443.

Clay, Z., de Waal, F.B.M., 2015. Sex and strife: post-conflict sexual contacts in bonobos. Behaviour 152, 313-334.

669 De Lathouwers, M., Van Elsacker, L., 2006. Comparing infant and juvenile behavior 670 in bonobos (Pan paniscus) and chimpanzees (Pan troglodytes): a preliminary study. Primates 47, 287-293. 
672 Dewaal, F.B.M., 1987. Tension Regulation and Nonreproductive Functions of Sex in Captive Bonobos (Pan-Paniscus). National Geographic Research 3, 318-335.

674 Durrleman, S., Pennec, X., Trouve, A., Ayache, N., Braga, J., 2012. Comparison of the endocranial ontogenies between chimpanzees and bonobos via temporal

Feldblum, J.T., Wroblewski, E.E., Rudicell, R.S., Hahn, B.H., Paiva, T., CetinkayaRundel, M., Pusey, A.E., Gilby, I.C., 2014. Sexually Coercive Male Chimpanzees Sire More Offspring. Current Biology 24, 2855-2860.

Furuichi, T., 2009. Factors underlying party size differences between chimpanzees and bonobos: a review and hypotheses for future study. Primates 50,197209.

Furuichi, T., Koops, K., Ryu, H., Sanz, C., Sakamaki, T., Morgan, D., Tokuyama, N., 2015. Why do wild bonobos not use tools like chimpanzees do? Behaviour $152,425-460$.

Garai, C., Furuichi, T., Kawamoto, Y., Ryu, H., Inoue-Murayama, M., 2014. Androgen receptor and monoamine oxidase polymorphism in wild bonobos. Meta Gene 2, 831-843.

Gariepy, J.L., Bauer, D.J., Cairns, R.B., 2001. Selective breeding for differential

696 Groves, C., 1981. (Comments) Bonobos: generalized hominid prototypes or specialized insular dwarfs? Current Anthropology 22, 363-375.

698 Gruber, T., Clay, Z., Zuberbuhler, K., 2010. A comparison of bonobo and chimpanzee tool use: evidence for a female bias in the Pan lineage. Animal Behaviour 80, 1023-1033.

701 Hammock, E.A.D., Young, L.J., 2005. Microsatellite instability generates diversity in brain and sociobehavioral traits. Science 308, 1630-1634. 
703 Haraway, D., 1993. Teddy Bear patriarchy: taxidermy in the Garden of Eden, New York City, 1908-36, Culture/Power/History. Princeton University Press, pp. 49-95.

Hare, B., 2007. From nonhuman to human mind - What changed and why? Current Directions in Psychological Science 16, 60-64.

Hare, B., 2009. What is the Effect of Affect on Bonobo and Chimpanzee Problem Solving? Neurobiology of Umwelt: How Living Beings Perceive the World, 89102.

Hare, B., 2011. From Hominoid to Hominid Mind: What Changed and Why? Annual Review of Anthropology, Vol 40 40, 293-309.

Hare, B., Call, J., Tomasello, M., 2001. Do chimpanzees know what conspecifics know? Animal Behaviour 61, 139-151.

Hare, B., Kwetuenda, S., 2010. Bonobos voluntarily share their own food with others. Current Biology 20, R230-R231.

Hare, B., Melis, A.P., Woods, V., Hastings, S., Wrangham, R., 2007. Tolerance allows bonobos to outperform chimpanzees on a cooperative task. Current Biology $17,619-623$.

Hare, B., Plyusnina, I., Ignacio, N., Schepina, O., Stepika, A., Wrangham, R., Trut, L., 2005. Social cognitive evolution in captive foxes is a correlated by-product of experimental domestication. Current Biology 15, 226-230. skilled than wolves with human social cues: a response to Udell et al.(2008) and Wynne, et al.(2008). Animal Behaviour 79, e1ee6.

Hare, B., Tomasello, M., 2005. Human-like social skills in dogs? Trends in Cognitive Sciences 9, 439-444.

Hare, B., Wobber, V., Wrangham, R., 2012. The self-domestication hypothesis: evolution of bonobo psychology is due to selection against aggression. Animal Behaviour 83, 573-585.

731 Hare, B., Yamamoto, S., 2015. Moving bonobos off the scientifically endangered list. Behaviour 152, 247-258. 
733 Hashimoto, C., Furuichi, T., 2006. Comparison of behavioral sequence of copulation between chimpanzees and bonobos. Primates 47, 51-55.

735 Haun, D.B.M., Nawroth, C., Call, J., 2011. Great Apes' Risk-Taking Strategies in a 736 Decision Making Task. Plos One 6.

737 Heilbronner, S.R., Rosati, A.G., Stevens, J.R., Hare, B., Hauser, M.D., 2008. A fruit in the 738 hand or two in the bush? Divergent risk preferences in chimpanzees and 739 bonobos. Biology Letters 4, 246-249.

740 Herrmann, E., Call, J., Hernandez-Lloreda, M.V., Hare, B., Tomasello, M., 2007. Humans have evolved specialized skills of social cognition: The cultural intelligence hypothesis. Science 317, 1360-1366.

Herrmann, E., Hare, B., Call, J., Tomasello, M., 2010. Differences in the Cognitive Skills of Bonobos and Chimpanzees. Plos One 5. temperament in nonhuman apes and human infants. Developmental Science $14,1393-1405$.

Herrmann, E., Keupp, S., Hare, B., Vaish, A., Tomasello, M., 2013. Direct and Indirect Reputation Formation in Nonhuman Great Apes (Pan paniscus, Pan troglodytes, Gorilla gorilla, Pongo pygmaeus) and Human Children (Homo sapiens). Journal of Comparative Psychology 127, 63-75.

Hill, K., Boesch, C., Goodall, J., Pusey, A., Williams, J., Wrangham, R., 2001. Mortality rates among wild chimpanzees. Journal of Human Evolution 40, 437-450.

Hohmann, G., 2001. Association and social interactions between strangers and residents in bonobos (Pan paniscus). Primates 42, 91-99.

Hohmann, G., Fruth, B., 2000. Use and function of genital contacts among female bonobos. Animal Behaviour 60, 107-120.

Hohmann, G., Fruth, B., 2002. Dynamics in social organization of bonobos (Pan paniscus), Behavioural Diversity in Chimpanzees and Bonobos. Cambridge University Press, pp. 138-150. species variation in behavior. Current Anthropology 44, 563-571. 
763 Hohmann, G., Fruth, B., 2003. Intra- and inter-sexual aggression by bonobos in the context of mating. Behaviour 140, 1389-1413.

Hohmann, G., Fruth, B., 2003. Lui Kotal: a new site for field research on bonobos in the Salonga National Park. Pan Africa News 10, 25-27.

Hopkins, W.D., Schaeffer, J., Russell, J.L., Bogart, S.L., Meguerditchian, A., Coulon, O., 2015. A comparative assessment of handedness and its potential neuroanatomical correlates in chimpanzees (Pan troglodytes) and bonobos (Pan paniscus). Behaviour 152, 461-492.

Idani, G., 1991. Social Relationships between Immigrant and Resident Bonobo (PanPaniscus) Females at Wamba. Folia Primatologica 57, 83-95.

Ihobe, H., 1992. Male-Male Relationships among Wild Bonobos (Pan-Paniscus) at Wamba, Republic-of-Zaire. Primates 33, 163-179.

Jaeggi, A.V., Stevens, J.M.G., Van Schaik, C.P., 2010. Tolerant Food Sharing and Reciprocity Is Precluded by Despotism Among Bonobos But Not Chimpanzees. American Journal of Physical Anthropology 143, 41-51.

Krupenye, C., Hare, B., (in prep). Bonobos prefer hinderers over helpers.

789 Krupenye, C., Rosati, A., Hare, B., (in press). Bonobos and chimpanzees exhibit economic framing effects. Biology Letters.

791 Kuroda, S., 1989. Developmental retardation and behavioral characteristics of pygmy chimpanzees. Understanding chimpanzees, 184-193. 
793

794

795

796

797

798

799

800

801

802

803

804

805

806

807

808

809

810

811

812

813

814

815

816

817

818

819

820

821

822

823

Lane, J.D., Wellman, H.M., Olson, S.L., Miller, A.L., Wang, L., Tardif, T., 2013. Relations Between Temperament and Theory of Mind Development in the United States and China: Biological and Behavioral Correlates of Preschoolers' FalseBelief Understanding. Developmental Psychology 49, 825-836.

Lane, J.D., Wellman, H.M., Olson, S.L., Miller, A.L., Wang, L., Tardif, T., 2013. Relations Between Temperament and Theory of Mind Development in the United States and China: Biological and Behavioral Correlates of Preschoolers' FalseBelief Understanding. Developmental Psychology 49, 825-836.

Leach, H.M., 2003. Human domestication reconsidered. Current Anthropology 44, 349-368.

Lewejohann, L., Pickel, T., Sachser, N., Kaiser, S., 2010. Wild genius - domestic fool? Spatial learning abilities of wild and domestic guinea pigs. Frontiers in Zoology 7.

Lieberman, D.E., Carlo, J., de Leon, M.P., Zollikofer, C.P.E., 2007. A geometric morphometric analysis of heterochrony in the cranium of chimpanzees and bonobos. Journal of Human Evolution 52, 647-662.

Liu, J.H., Portnoy, J., Raine, A., 2012. Association between a marker for prenatal testosterone exposure and externalizing behavior problems in children. Development and Psychopathology 24, 771-782.

MacLean, E., Hare, B., 2013. Spontaneous triadic engagement in bonobos (Pan paniscus) and chimpanzees (Pan troglodytes). Journal of Comparative Psychology 127, 245.

MacLean, E.L., Hare, B., 2012. Bonobos and chimpanzees infer the target of another's attention. Animal Behaviour 83, 345-353.

MacLean, E.L., Hare, B., 2015. Bonobos and chimpanzees exploit helpful but not prohibitive gestures. Behaviour 152, 493-520.

MacLean, E.L., Hare, B., 2015. Dogs hijack the human bonding pathway. Science 348, 280-281.

MacLean, E.L., Hare, B., Nunn, C.L., Addessi, E., Amici, F., Anderson, R.C., Aureli, F., Baker, J.M., Bania, A.E., Barnard, A.M., Boogert, N.J., Brannon, E.M., Bray, E.E., Bray, J., Brent, L.J.N., Burkart, J.M., Call, J., Cantlon, J.F., Cheke, L.G., Clayton, 
N.S., Delgado, M.M., DiVincenti, L.J., Fujita, K., Herrmann, E., Hiramatsu, C., Jacobs, L.F., Jordan, K.E., Laude, J.R., Leimgruber, K.L., Messer, E.J.E., Moura, A.C.D., Ostojic, L., Picard, A., Platt, M.L., Plotnik, J.M., Range, F., Reader, S.M., Reddy, R.B., Sandel, A.A., Santos, L.R., Schumann, K., Seed, A.M., Sewall, K.B., Shaw, R.C., Slocombe, K.E., Su, Y.J., Takimoto, A., Tan, J.Z., Tao, R., van Schaik, C.P., Viranyi, Z., Visalberghi, E., Wade, J.C., Watanabe, A., Widness, J., Young, J.K., Zentall, T.R., Zhao, Y.N., 2014. The evolution of self-control. Proceedings of the National Academy of Sciences of the United States of America 111, E2140-E2148.

MacLean, E.L., Matthews, L.J., Hare, B.A., Nunn, C.L., Anderson, R.C., Aureli, F., Brannon, E.M., Call, J., Drea, C.M., Emery, N.J., Haun, D.B.M., Herrmann, E., Jacobs, L.F., Platt, M.L., Rosati, A.G., Sandel, A.A., Schroepfer, K.K., Seed, A.M., Tan, J.Z., van Schaik, C.P., Wobber, V., 2012. How does cognition evolve? Phylogenetic comparative psychology. Animal Cognition 15, 223-238. Mailund, T., Halager, A.E., Westergaard, M., Dutheil, J.Y., Munch, K., Andersen, L.N., Lunter, G., Prufer, K., Scally, A., Hobolth, A., Schierup, M.H., 2012. A New Isolation with Migration Model along Complete Genomes Infers Very Different Divergence Processes among Closely Related Great Ape Species. Plos Genetics 8.

McIntyre, M.H., Herrmann, E., Wobber, V., Halbwax, M., Mohamba, C., de Sousa, N., Atencia, R., Cox, D., Hare, B., 2009. Bonobos have a more human-like secondto-fourth finger length ratio (2D:4D) than chimpanzees: a hypothesized indication of lower prenatal androgens. Journal of Human Evolution 56, 361365.

Melis, A.P., Warneken, F., Hare, B., 2010. Collaboration and helping in chimpanzees. The mind of the chimpanzee: ecological and experimental perspectives, 278393.

Micheletta, J., Waller, B.M., 2012. Friendship affects gaze following in a tolerant species of macaque, Macaca nigra. Animal Behaviour 83, 459-467. 
853 Miklosi, A., Kubinyi, E., Topal, J., Gacsi, M., Viranyi, Z., Csanyi, V., 2003. A simple

854 reason for a big difference: Wolves do not look back at humans, but dogs do.

$855 \quad$ Current Biology 13, 763-766.

856 Morimoto, N., de Leon, M.S.P., Zollikofer, C.P.E., 2014. Phenotypic Variation in

857 Infants, Not Adults, Reflects Genotypic Variation among Chimpanzees and

$858 \quad$ Bonobos. Plos One 9.

859 Moscovice, L.R., Deschner, T., Hohmann, G., 2015. Welcome Back: Responses of

860 Female Bonobos (Pan paniscus) to Fusions. Plos One 10.

861 Muller, M.N., Kahlenberg, S.M., Thompson, M.E., Wrangham, R.W., 2007. Male

862 coercion and the costs of promiscuous mating for female chimpanzees.

863 Proceedings of the Royal Society B-Biological Sciences 274, 1009-1014.

864 Muller, M.N., Mitani, J.C., 2005. Conflict and cooperation in wild chimpanzees.

865 Advances in the Study of Behavior, Vol 35 35, 275-331.

866 Muller, M.N., Thompson, M.E., Kahlenberg, S.M., Wrangham, R.W., 2011. Sexual

867 coercion by male chimpanzees shows that female choice may be more

868 apparent than real. Behavioral Ecology and Sociobiology 65, 921-933.

869 Nagasawa, M., Kikusui, T., Onaka, T., Ohta, M., 2009. Dog's gaze at its owner

870 increases owner's urinary oxytocin during social interaction. Hormones and

871 Behavior 55, 434-441.

872 Nagasawa, M., Mitsui, S., En, S., Ohtani, N., Ohta, M., Sakuma, Y., Onaka, T., Mogi, K.,

873 Kikusui, T., 2015. Oxytocin-Gaze Positive Loop and the Coevolution of

874 Human-Dog Bonds. Obstetrical \& Gynecological Survey 70, 450-451.

875 Nelson, E., Rolian, C., Cashmore, L., Shultz, S., 2011. Digit ratios predict polygyny in

876 early apes, Ardipithecus, Neanderthals and early modern humans but not in

877 Australopithecus. Proceedings of the Royal Society B-Biological Sciences 278,

$878 \quad$ 1556-1563.

879 Oliva, J.L., Rault, J.L., Appleton, B., Lill, A., 2015. Oxytocin enhances the appropriate

880 use of human social cues by the domestic dog (Canis familiaris) in an object

881 choice task (vol 18, pg 767, 2015). Animal Cognition 18, 991-991.

882 Palagi, E., Cordoni, G., 2012. The right time to happen: play developmental

883 divergence in the two Pan species. 
884 Palagi, E., Paoli, T., 2007. Play in adult Bonobos (Pan paniscus): Modality and potential meaning. American Journal of Physical Anthropology 134, 219-225.

886 Paoli, T., Tacconi, G., Tarli, S.M.B., Palagi, E., 2007. Influence of feeding and shortterm crowding on the sexual repertoire of captive bonobos (Pan paniscus). Annales Zoologici Fennici 44, 81-88.

Petit, O., Desportes, C., Thierry, B., 1992. Differential Probability of Coproduction in 2 Species of Macaque (Macaca-Tonkeana, M-Mulatta). Ethology 90, 107-120. G., Kodira, C., Winer, R., 2012. The bonobo genome compared with the chimpanzee and human genomes. Nature 486, 527-531.

Pusey, A., Murray, C., Wallauer, W., Wilson, M., Wroblewski, E., Goodall, J., 2008. Severe aggression among female Pan troglodytes schweinfurthii at Gombe National Park, Tanzania. International Journal of Primatology 29, 949-973.

Pusey, A.E., Schroepfer-Walker, K., 2013. Female competition in chimpanzees. Philosophical Transactions of the Royal Society B-Biological Sciences 368.

Rodrigues, S.M., Saslow, L.R., Garcia, N., John, O.P., Keltner, D., 2009. Oxytocin receptor genetic variation relates to empathy and stress reactivity in humans. Proceedings of the National Academy of Sciences of the United States of America 106, 21437-21441.

Romero, T., Nagasawa, M., Mogi, K., Hasegawa, T., Kikusui, T., 2014. Oxytocin promotes social bonding in dogs. Proceedings of the National Academy of Sciences of the United States of America 111, 9085-9090.

Rosati, A.G., Hare, B., 2012. Chimpanzees and bonobos exhibit divergent spatial memory development. Developmental Science 15, 840-853.

Rosati, A.G., Hare, B., 2012. Decision making across social contexts: competition increases preferences for risk in chimpanzees and bonobos. Animal Behaviour 84, 869-879.

Rosati, A.G., Hare, B., 2013. Chimpanzees and Bonobos Exhibit Emotional Responses to Decision Outcomes. Plos One 8.

Rosati, A.G., Rodriguez, K., Hare, B., 2014. The ecology of spatial memory in four lemur species. Animal Cognition 17, 947-961. 
915 Rosati, A.G., Stevens, J.R., Hare, B., Hauser, M.D., 2007. The evolutionary origins of human patience: Temporal preferences in chimpanzees, bonobos, and human adults. Current Biology 17, 1663-1668.

918 Rosati, A.G., Stevens, J.R., Hauser, M.D., 2006. The effect of handling time on temporal 919 discounting in two New World primates. Animal Behaviour 71, 1379-1387.

920 Ryu, H., Hill, D.A., Furuichi, T., 2015. Prolonged maximal sexual swelling in wild bonobos facilitates affiliative interactions between females. Behaviour 152, 285-311.

Sakamaki, T., Behncke, I., Laporte, M., Mulavwa, M., Ryu, H., Takemoto, H., Tokuyama, N., Yamamoto, S., Furuichi, T., 2015. Intergroup Transfer of Females and Social Relationships Between Immigrants and Residents in Bonobo (Pan paniscus) Societies, Dispersing Primate Females. Springer, pp. 127-164.

Santos, L.R., Rosati, A.G., 2015. The Evolutionary Roots of Human Decision Making. Annual Review of Psychology, Vol 66 66, 321-347.

Schroepfer-Walker, K., Wobber, V., Hare, B., 2015. Experimental evidence that grooming and play are social currency in bonobos and chimpanzees. Behaviour 152, 545-562.

Schubert, G., Vigilant, L., Boesch, C., Klenke, R., Langergraber, K., Mundry, R., Surbeck, M., Hohmann, G., 2013. Co-Residence between Males and Their Mothers and Grandmothers Is More Frequent in Bonobos Than Chimpanzees. Plos One 8.

Schultz, A.H., 1941. The Relative Size of the Cranial Capacity in Primates. American Journal of Physical Anthropology 28, 273-287.

Serckx, A., Kuhl, H.S., Beudels-Jamar, R.C., Poncin, P., Bastin, J.F., Huynen, M.C., 2015. Feeding Ecology of Bonobos Living in Forest-Savannah Mosaics: Diet Seasonal Variation and Importance of Fallback Foods. American Journal of Primatology 77, 948-962.

Somel, M., Franz, H., Yan, Z., Lorenc, A., Guo, S., Giger, T., Kelso, J., Nickel, B., Dannemann, M., Bahn, S., Webster, M.J., Weickert, C.S., Lachmann, M., Paabo, S., Khaitovich, P., 2009. Transcriptional neoteny in the human brain. 
Proceedings of the National Academy of Sciences of the United States of America 106, 5743-5748.

947 Sommer, V., Bauer, J., Fowler, A., Ortmann, S., 2011. Patriarchal Chimpanzees, Matriarchal Bonobos: Potential Ecological Causes of a Pan Dichotomy.

Staes, N., Stevens, J.M.G., Helsen, P., Hillyer, M., Korody, M., Eens, M., 2014. Oxytocin

Stevens, J.M.G., de Groot, E., Staes, N., 2015. Relationship quality in captive bonobo and Vasopressin Receptor Gene Variation as a Proximate Base for Inter- and Intraspecific Behavioral Differences in Bonobos and Chimpanzees. Plos One groups. Behaviour 152, 259-283.

Surbeck, M., Hohmann, G., 2008. Primate hunting by bonobos at LuiKotale, Salonga National Park. Current Biology 18, R906-R907.

Surbeck, M., Mundry, R., Hohmann, G., 2011. Mothers matter! Maternal support, dominance status and mating success in male bonobos (Pan paniscus). Proceedings of the Royal Society B-Biological Sciences 278, 590-598.

963 Tan, J., Kwetuenda, S., Hare, B., 2015. Preference or paradigm? Bonobos show no evidence of other-regard in the standard prosocial choice task. Behaviour

966 Tan, J., Hare, B., 2013. Bonobos Share with Strangers. Plos One 8.

967 Thompson, J.A.M., 2003. A model of the biogeographical journey from Proto-pan to 968 Pan paniscus. Primates 44, 191-197.

969 Tomasello, M., Hare, B., Lehmann, H., Call, J., 2007. Reliance on head versus eyes in 970 the gaze following of great apes and human infants: the cooperative eye 971 hypothesis. Journal of Human Evolution 52, 314-320.

972 Topal, J., Gergely, G., Erdohegyi, A., Csibra, G., Miklosi, A., 2009. Differential 973 Sensitivity to Human Communication in Dogs, Wolves, and Human Infants. $974 \quad$ Science 325, 1269-1272. 
975 Trut, L., Oskina, I., Kharlamova, A., 2009. Animal evolution during domestication: the domesticated fox as a model. Bioessays 31, 349-360.

977 Trut, L.N., 1999. Early canid domestication: The farm-fox experiment. American Scientist 87, 160-169.

Vlamings, P.H.J.M., Hare, B., Call, J., 2010. Reaching around barriers: the performance of the great apes and 3-5-year-old children. Animal Cognition 13, 273-285.

Waal, F.B., Lanting, F., 1997. Bonobo: The forgotten ape. University of California Press.

Warneken, F., Hare, B., Melis, A.P., Hanus, D., Tomasello, M., 2007. Spontaneous altruism by chimpanzees and young children. Plos Biology 5, 1414-1420.

Watts, D.P., Mitani, J.C., 2002. Hunting behavior of chimpanzees at Ngogo, Kibale National Park, Uganda. International Journal of Primatology 23, 1-28.

Wellman, H.M., Lane, J.D., LaBounty, J., Olson, S.L., 2011. Observant, nonaggressive temperament predicts theory-of-mind development. Developmental Science 14, 319-326.

West-Eberhard, M.J., 2003. Developmental plasticity and evolution. Oxford University Press.

Wilkins, A.S., Wrangham, R.W., Fitch, W.T., 2014. The "Domestication Syndrome" in Mammals: A Unified Explanation Based on Neural Crest Cell Behavior and Genetics. Genetics 197, 795-808.

Wittig, R.M., Crockford, C., Deschner, T., Langergraber, K.E., Ziegler, T.E., bonding in related and unrelated wild chimpanzees. Proceedings of the Royal Society B-Biological Sciences 281. 
1006 Wobber, V., Hare, B., 2011. Psychological Health of Orphan Bonobos and Chimpanzees in African Sanctuaries. Plos One 6.

Wobber, V., Hare, B., Maboto, J., Lipson, S., Wrangham, R., Ellison, P., 2010. Differential reactivity of steroid hormones in chimpanzees and bonobos when anticipating food competition. Proceedings of the National Academy of Sciences, USA 107, 12457-12462.

Wobber, V., Herrmann, E., 2015. The influence of testosterone on cognitive 1013 performance in bonobos and chimpanzees. Behaviour 152, 407-423.

Wobber, V., Wrangham, R., Hare, B., 2010. Application of the heterochrony

Wobber, V., Wrangham, R., Hare, B., 2010. Bonobos exhibit delayed development of social behavior and cognition relative to chimpanzees. Current Biology 20, 226-230.

Woods, V., Hare, B., 2011. Bonobo but not chimpanzee infants use socio-sexual contact with peers. Primates 52, 111-116.

Wrangham, R., Pilbeam, D., 2001. African apes as time machines. All Apes Great and Small, Vol 1: African Apes, 5-17.

Wrangham, R.W., 1993. The Evolution of Sexuality in Chimpanzees and Bonobos. Human Nature-an Interdisciplinary Biosocial Perspective 4, 47-79. Wrangham, R.W., 1999. Evolution of coalitionary killing. Yearbook of Physical Anthropology 1999, Vol 42 42, 1-30.

1031 Wrangham, R., 2002. The cost of sexual attraction: is there a trade-off in female Pan 1032 between sex appeal and received coercion?, Behavioral Diversity in Chimpanzees and Bonobos. Cambridge University Press, pp. 204-215.

1034 Wrangham, R.W., 2014. 16. Ecology and Social Relationships in Two Species of 1035 Chimpanzee. Ecological aspects of social evolution: Birds and mammals, 352. 
1036 Wrangham, R.W., Peterson, D., 1997. Demonic males: Apes and the origins of human violence. Houghton Mifflin Harcourt.

1038 Wrangham, R.W., Wilson, M.L., Muller, M.N., 2006. Comparative rates of violence in 1039 chimpanzees and humans. Primates 47, 14-26.

1040 Yamamoto, S., 2015. Non-reciprocal but peaceful fruit sharing in wild bonobos in 1041 Wamba. Behaviour 152, 335-357.

1042 Yamamoto, S., Humle, T., Tanaka, M., 2012. Chimpanzees' flexible targeted helping 1043 based on an understanding of conspecifics' goals. Proceedings of the National 1044 Academy of Sciences of the United States of America 109, 3588-3592.

1045 Zihlman, A.L., Cramer, D.L., 1978. Skeletal Differences between Pygmy (Pan1046 Paniscus) and Common Chimpanzees (Pan-Troglodytes). Folia Primatologica $1047 \quad 29,86-94$.

1048 


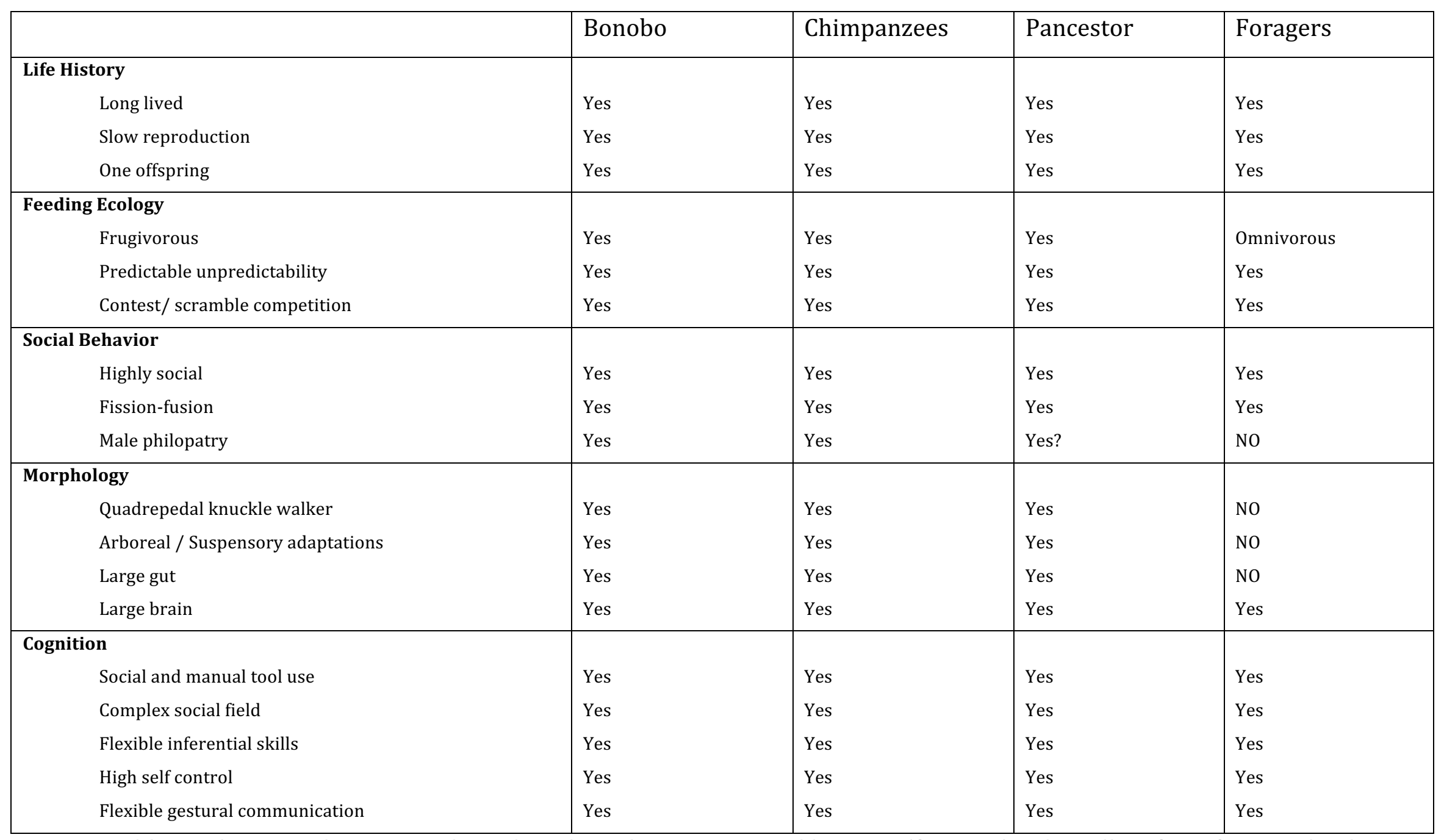

Table 1. The traits that are similar in bonobos, chimpanzees and humans (foragers). They allow for inference that humans inherited these traits through common descent and their origin is ancient. The traits that clearly differ in humans are likely derived in human lineage since all other apes seem to share them. 


\section{Bonobo Chimpanzee}

Human

Foragers

Extractive foraging $^{{ }^{* *}}$

Non-conceptive sexual behaviour ${ }^{2}$

Lethal aggression between groups ${ }^{3}$

Only captivity Frequent Frequent

Mother's importance to adult offspring ${ }^{4}$

Infanticide/Female Coercion ${ }^{5}$

\begin{tabular}{c|c|c|} 
Frequent & Absent & Frequent \\
\hline Absent & Present & Present
\end{tabular}

High Low

High

\begin{tabular}{|c|c|c|}
\hline Absent & Present & Present \\
\hline High & Low & High \\
\hline Absent & Present & Present \\
\hline Present & Absent & Present \\
\hline Absent & Frequent & Frequent \\
\hline High & Low & High \\
\hline
\end{tabular}

Levels of adult play ${ }^{6}$

Cooperative hunting ${ }^{7}$

Sharing between strangers ${ }^{8}{ }^{*}$

Male-male alliances ${ }^{9}$

Female gregariousness ${ }^{10}$ *

1050 Table 2. Behaviours in bonobos and chimpanzees more similar to humans than 1051 each other with * indicating each relevant paper from this special issue:

1052 1Hohmann \& Fruth, 2003a; Gruber et al, 2010; *Furuichi et al; 2015; *Hopkins et 1053 al 2015; ${ }^{2}$ Kano, 1992, Hashimoto \& Furuichi, 2006; Hohmann \& Fruth, 2000; 
1054 Hare et al, 2007; Hare \& Woods, 2011; *Ryu et al, 2015; *Clay \& deWaal, 2015, 1055 3Wrangham, 1999; Wilson et al, 2014; ${ }^{4}$ De Lathouwers \& Van Elsacker, 2006; 1056 Surbeck et al 2011; Schubert et al 2013; ${ }^{5}$ Hohmann \& Fruth, 2002; Surbeck et al 1057 2011: ${ }^{6}$ Palagi \& Paoli, 2007, Wobber et al, 2010; ${ }^{7}$ Ihobe, 1993; Mitani \& Watts, 1058 2001; Surbeck \& Hohmann, 2008; ${ }^{8}$ Yamamoto et al., in prep. Tan \& Hare, 2013;

$1059 \quad *$ Tan et al, 2015; ${ }^{9}$ Kano, 1992, Wrangham, 1999; ${ }^{10}$ Furuichi, 2011; *Stevens et al, 1060 2015. Even with the central role bonobos can play in testing hypothesis 1061 regarding ape and human evolution, bonobo research lags far behind work with 1062 chimpanzees. Searching ISI Web of Science and Google Scholar for "bonobo" and 1063 "chimpanzee" reveals that the bonobo makes up only $3 \%$ and $9 \%$ of the total 1064 citations indexed for both species collectively. This is far from the 50\% that 1065 phylogeny alone would predict (Republished from Hare \& Yamamoto, 2015). 


\begin{tabular}{|c|c|c|}
\hline Juvenilized Traits in Bonobos & $\begin{array}{l}\text { Descriptions of delayed development in } \\
\text { comparison to chimpanzees: }\end{array}$ & References \\
\hline Play behavior & $\begin{array}{l}\text { Adult Bonobos show juvenile levels of play in as } \\
\text { adults }\end{array}$ & $\begin{array}{l}\text { Palagi \& Paoli, 2007; } \\
\text { Hare et al, 2007; Wobber } \\
\text { et al, 2010; Palagi \& } \\
\text { Cordoni, } 2012\end{array}$ \\
\hline $\begin{array}{l}\text { Non-conceptive sexual } \\
\text { behavior }\end{array}$ & $\begin{array}{l}\text { Adult bonobos show higher levels of } \\
\text { nonconceptive social sexual behavior than } \\
\text { chimpanzees* }\end{array}$ & $\begin{array}{l}\text { Hare et al, } 2007 \\
\text { *Although see Wood \& } \\
\text { Hare, } 2011\end{array}$ \\
\hline Xenophilia and Prosociality & $\begin{array}{l}\text { Adult bonobos show juvenile levels of attraction } \\
\text { to strangers (i.e. similar to immigrant females). } \\
\text { Are prosocial not aggressive toward strangers in } \\
\text { dyadic interactions. }\end{array}$ & $\begin{array}{l}\text { Hare \& Kwetuenda, } \\
\text { 2010; Tan et al, } 2013\end{array}$ \\
\hline Maternal dependence & $\begin{array}{l}\text { Bonobos infants remain dependent on mother } \\
\text { for longer; Adult males remain socially } \\
\text { dependent on mothers throughout life. }\end{array}$ & $\begin{array}{l}\text { De Lathouwers \& Van } \\
\text { Elsacker, 2006; Surbeck } \\
\text { et al, } 2011\end{array}$ \\
\hline Food Sharing & $\begin{array}{l}\text { Adult bonobos show juvenile levels of food } \\
\text { sharing observed in chimpanzees. }\end{array}$ & $\begin{array}{l}\text { Hare et al, 2007; } \\
\text { Wobber et al, } 2010\end{array}$ \\
\hline Social inhibition & $\begin{array}{l}\text { Bonobo infants and juveniles show delays in } \\
\text { development of social inhibition. }\end{array}$ & Wobber et al, 2010 \\
\hline Spatial memory & $\begin{array}{l}\text { Bonobos show delayed development in their } \\
\text { ability to remember locations of hidden food. }\end{array}$ & Rosati \& Hare, 2012 \\
\hline Physical cognition & $\begin{array}{l}\text { Bonobos show delayed development in } \\
\text { understanding of the physical properties of the } \\
\text { world (i.e. connectivity, transposition, etc.). }\end{array}$ & Wobber et al, 2014 \\
\hline
\end{tabular}

1067 Table 3. Cognitive and behavioral traits for which bonobos have been found to be

1068 juvenilized relative to chimpanzees and the relevant citation for quantitative

1069 comparisons that demonstrate delayed development in bonobos. 